\title{
Seedling growth and development of Prosopis juliflora (Sw.) DC. in different soil types of industrial and non- industrial areas
}

\author{
Muhammad Kabir ${ }^{1}$, Muhammad Zafar Iqbal $^{2}$, Muhammad Shafiq ${ }^{2^{*}}$, Zia-ur-Rehman Farooqi ${ }^{2}$ \\ and Um E Habiba ${ }^{3}$
}

${ }^{1}$ Deparment of Biological Sciences, University of Sargodha, Sub-Campus Bhakkar, Bhakkar, Pakistan.

${ }^{2}$ Department of Botany, University of Karachi, Karachi, 75270, Pakistan.

${ }^{3}$ Department of Physics, Riphah International University, Faisalabad Campus, Pakistan.

Accepted 4 February, 2020

\begin{abstract}
The problem of environmental pollution has been growing rapidly for the last couple of years as a result of discharge of various kinds of pollutants from unplanned industrialization, particularly in developing countries and affecting air, water soil and vegetation. The effects of different soil types on seedling growth performance of Prosopis juliflora (Sw.) DC. were recorded. Seedlings of $P$. julliflora which were grown in soils of Indus Battery Factory, Universal Chemicals Factory, Haroon Textile Factory and National Foods Ltd. Factory showed significant $(p<0.05)$ variation in growth variables like root, shoot, seedling length, plant cover, leaf area, root, shoot and leaf dry weight and total plant dry weight of $P$. julliflora as compared to plants grown in soil of control area (Karachi University Campus). It was concluded that the seedlings of $P$. julliflora showed significantly $(\mathrm{p}<0.05)$ more root, shoot, seedling length, plant cover and leaf area in Haroon Textile Factory soil compared to Indus Battery Factory and Universal Chemicals Factory soil treatment. The root, shoot, leaf and total plant dry weight of $P$. julliflora was also decreased in these industrial areas soil as compared to Karachi University Campus soil. Soil of Haroon Textile Factory promoted shoot and seedling length of $P$. julliflora relative to the seedlings of $P$. julliflora grown in control soil (Karachi University soil).
\end{abstract}

Keywords: Biomass production, seedling growth, soil pollution, soils types.

*Corresponding author. E-mail address: shafiqeco@yahoo.com.

\section{INTRODUCTION}

Karachi is the major commercial center of Pakistan situated in Sindh province, which presently has a population of more than twenty million. In order to fulfill the needs of different types of products for these people and export, industrial activities are increasing unplanned. The activities of these industries are responsible for polluting the environment due to discharge of waste in air, water and soil without any pretreatment. Ultimately, pollution stress affect is being forced upon human beings and other living organisms. Karachi city has five main industrial estates, that is, Sindh Industrial Treading Estate (S.I.T.E.), Korangi Industrial Treading Estate (K.I.T.E.) and Landhi Industrial Treading Estate (L.I.T.E.), North Karachi Industrial Treading Estate (N.I.T.E.) and Hub Industrial Treading Estate (H.I.T.E.). Sindh Industrial Treading Estate (S.I.T.E.) is a densely populated town in the western part of Karachi, Pakistan. The town is bordered by Gadap Town to the North, Liquatabad and North Nazimabad to the east across the Orangi Nala stream, Layari and Saddar to the South across the Layari River and Kiamari to the West. It is the oldest and the largest designated industrial area of Pakistan, encompassing 4700 acres $\left(19 \mathrm{~km}^{2}\right)$ area and contains approximately 2,400 factories which are discharging their 
wastes into surrounding, thus causing environment pollution.

Problem of environmental pollution has existed for centuries and is still growing rapidly as a result of unplanned industrialization, particularly in developing countries (Naheed et al., 1986; Rehman, 2006; Rehman and Iqbal, 2006; Shafiq and lqbal, 2012; Shafiq et al., 2019). The hazard and fast industrial growth are causing an enormous environmental pollution affecting plants and human being. Abdel and Aly (2000) indicated that soils of industrial area showed significant accumulation of heavy metals reducing the plants growth. Heavy metals contamination affects the biosphere in many places worldwide (Cunningham et al., 1997; Meagher, 2000; Raskin and Ensley, 2000). Excess concentrations of some heavy metals in soil such as $\mathrm{Cd}$ (II), $\mathrm{Cr}$ (VI), Ni (II) and $\mathrm{Zn}$ (II), agrochemicals have caused the disruption of natural, aquatic terrestrial ecosystems and agriculture (Leyval et al., 1995; Gardea-Torresdey et al., 1996; Meagher, 2000; Millar et al., 2000).

Prosopis juliflora (Sw.) DC. is an evergreen tree native to South America, Central America, the Caribbean and belongs to family Mimosaceae. It can grow very fast even in polluted soil, tolerant to arid conditions and saline soils. $P$. juliflora produced yellow flowers and pods which are green in colour when immature turned yellow when they mature contain a high level of sugar and are palatable to livestock when ripe (Masilamani and Vadivelu, 1997; Talpada and Shukla, 1988; Alban et al., 2002; Batista et al., 2002). As $P$. juliflora is able to improve the soil in which it is growing by means of biological nitrogen fixation, leaf litter accumulation, nutrient obtaining from deeper soil layers, loosening of a hard soil structure, stabilizing of loose sands, and an increase of the fauna above and below the ground and help in preventing soil erosion (Kaushik and Kumar, 2003; Lenacuru, 2003). P. juliflora significantly serves as good fuel wood source and helps in controlling soil erosion.

The different types of industries include, garments, bones crushing, coloring, metal processing, pharmaceutical, textile, chemicals, food processing and battery manufacturing industries are present in the S.I.T.E. (Sindh Industrial Treading Estate) areas of Karachi, Pakistan. These industries discharge different kinds of waste in air, water and soil which are especially, polluting the soil of nearby places, thus causing positive and negative effects to plant growing around the industrial areas.

$P$. juliflora is widely distributed in different regions of Pakistan. $P$. juliflora has the ability to thrive in harsh climatic condition and stabilize the soil texture and structure in semi-arid region. There are many evidences available on the impact of industrial activities on soil types on plant growth. In view of the different role of industrial activities of Karachi, it is necessary to investigate the effects of soil of industrial areas on growth of different plant species. In the present study the effects of different soil types of industrial and non-industrial areas on seedling growth of $P$. juliflora was carried out.

\section{MATERIALS AND METHODS}

The experiment was conducted in green house under the uniform natural environmental conditions at the Department of Botany, in the University of Karachi, Pakistan. The physico-chemical characteristics of the soil of area were studied earlier (Kabir, 2014). Healthy and uniform size seeds of $P$. juliflora were collected from the University of Karachi Campus. The top ends of the seeds were slightly cut with clean scissors to break dormancy and were sown in garden soil at $1 \mathrm{~cm}$ depth of soil in earthen pots and watered regularly. After two weeks of their germination, uniform size seedlings were transplanted in plastic pots of $20 \mathrm{~cm}$ in diameter and $9.8 \mathrm{~cm}$ in depth containing the soil of University Campus as control and industrial polluted soil. There were five replicates for each treatment. The experiment was completely randomized. Only one seedling was grown in each pot and the plants were treated with tap water regularly. Every week reshuffling of pots were also carried to avoid light/ shade or any other green house effects. Seedling height, number of leaves, leaf area and plant circumference were noted after every week. After eight weeks seedlings were taken out from pots, their roots washed with water; and root length, shoot length, seedlings length (which included length of root and shoot), plant circumference, number of leaves and leaf area were measured. Seedlings were dried in an oven at $80^{\circ} \mathrm{C}$ for $24 \mathrm{~h}$ and oven dry weights $(\mathrm{g})$ of seedlings were determined.

Percent reduction or promotion in different growth parameters of the plants grown in industrial soils of polluted sites was determined in comparison with a control site using the following formula (Grigalaviciene et al., 2005).

$$
\% \text { Promotion } / \text { Reduction }=\frac{\text { Control }- \text { Treatment }}{\text { Control }} \times 100
$$

\section{Statistical analysis}

Data of different growth parameters were statistically analyzed by ANOVA and Duncan Multiple Range Test on personal computer at $p<0.05$ level. Pearson correlation was applied to investigate relationship between different soil characteristics and growth parameters of plants using the SPSS software.

\section{RESULTS}

The results of the present studies showed that the use of soils types of industrial and nonindustrial areas showed different impact on seedling growth performance of an important tree species, P. juliflora (Table 1a-b, 2 to 7 , Figures 1 and 2). The soil treatment of Haroon Textile Factory showed significantly $(p<0.05)$ highest root $(20.33 \mathrm{~cm})$, shoot $(30.24 \mathrm{~cm})$, seedling length $(50.56 \mathrm{~cm})$, plant cover $(28.68 \mathrm{~cm})$, number of leaves $(72.40 \mathrm{~cm})$ and leaf area (3.32 sq. $\mathrm{cm}$ ) of $P$. juliflora as compared to other soil treatments (Table 1a). The seedlings of $P$. juliflora grown in Indus Battery Factory showed significantly $(\mathrm{p}<$ $0.05)$ lowest root $(8.80 \mathrm{~cm})$, shoot $(9.76 \mathrm{~cm})$, seedling length $(17.46 \mathrm{~cm})$, plant cover $(14.22 \mathrm{~cm})$, number of leaves (13.20) and leaf area (0.79 sq. $\mathrm{cm})$ as compared to control. A better growth parameter as root, shoot and seedling length, plant cover, number of leaves, leaf area and biomass variables as seedling fresh weight, root, 
Table 1a. Growth of Prosopis juliflora in soils of different areas.

\begin{tabular}{lcccccc}
\hline Treatments & $\begin{array}{c}\text { Root length } \\
(\mathbf{c m})\end{array}$ & $\begin{array}{c}\text { Shoot length } \\
(\mathbf{c m})\end{array}$ & $\begin{array}{c}\text { Seedling length } \\
(\mathbf{c m})\end{array}$ & $\begin{array}{c}\text { Plant cover } \\
(\mathbf{c m})\end{array}$ & Number of leaves & $\begin{array}{c}\text { Leaf area } \\
(\mathbf{S q} \mathbf{~ c m})\end{array}$ \\
\hline $\mathrm{A}$ & $14.54 \pm 2.96^{\mathrm{b}}$ & $14.40 \pm 1.84^{\mathrm{b}}$ & $28.94 \pm 4.34^{\mathrm{bc}}$ & $16.94 \pm 0.18^{\mathrm{c}}$ & $27.80 \pm 4.02^{\mathrm{bc}}$ & $1.18 \pm 0.19^{\mathrm{ab}}$ \\
$\mathrm{B}$ & $08.08 \pm 0.66^{\mathrm{c}}$ & $09.76 \pm 1.24^{\mathrm{b}}$ & $17.46 \pm 1.59^{\mathrm{a}}$ & $14.22 \pm 0.19^{\mathrm{d}}$ & $13.20 \pm 2.58^{\mathrm{c}}$ & $0.79 \pm 0.12^{\mathrm{b}}$ \\
$\mathrm{C}$ & $13.20 \pm 1.78^{\mathrm{bc}}$ & $15.08 \pm 1.99^{\mathrm{b}}$ & $28.28 \pm 3.66^{\mathrm{bc}}$ & $16.56 \pm 0.23^{\mathrm{c}}$ & $27.20 \pm 5.17^{\mathrm{bc}}$ & $1.69 \pm 0.30^{\mathrm{ab}}$ \\
$\mathrm{D}$ & $20.33 \pm 2.32^{\mathrm{a}}$ & $30.24 \pm 3.67^{\mathrm{a}}$ & $50.56 \pm 3.88^{\mathrm{a}}$ & $28.68 \pm 0.78^{\mathrm{a}}$ & $72.40 \pm 10.37^{\mathrm{a}}$ & $3.32 \pm 0.28^{\mathrm{ab}}$ \\
$\mathrm{E}$ & $14.12 \pm 1.19^{\mathrm{b}}$ & $25.36 \pm 4.71^{\mathrm{a}}$ & $39.48 \pm 5.55^{\mathrm{ab}}$ & $27.02 \pm 0.32^{\mathrm{b}}$ & $53.00 \pm 14.09^{\mathrm{ab}}$ & $2.38 \pm 0.39^{\mathrm{a}}$ \\
L.S.D. $($ p $<0.05)$ & 5.54 & 8.81 & 11.84 & 1.20 & 24.89 & 4.69 \\
\hline
\end{tabular}

Sites: $A=$ Karachi University Campus; $B=$ Indus Battery Factory; $C=$ Universal Chemicals factory; $D=$ Haroon Textile factory; $E=$ National Foods Ltd. Factory. Means followed by the same letter in the same column are not significantly different according to Duncan Multiple Range Test at $p<0.05$ level. \pm Standard Error, L.S.D. Least Significant Difference.

Table 1b. Seedling fresh, dry weights and ratios of different variables of Prosopis juliflora in soils of different areas.

\begin{tabular}{lccccc}
\hline Treatments & $\begin{array}{c}\text { Seedling fresh } \\
\text { weight }(\mathbf{g})\end{array}$ & $\begin{array}{c}\text { Root dry weight } \\
(\mathbf{g})\end{array}$ & $\begin{array}{c}\text { Shoot dry weight } \\
(\mathbf{g})\end{array}$ & $\begin{array}{c}\text { Leaf dry weight } \\
(\mathbf{g})\end{array}$ & $\begin{array}{c}\text { Total plant dry } \\
\text { weight }(\mathbf{g})\end{array}$ \\
\hline A & $0.73 \pm 0.06^{\mathrm{c}}$ & $0.08 \pm 0.01^{\mathrm{c}}$ & $0.16 \pm 0.02^{\mathrm{c}}$ & $0.11 \pm 0.01^{\mathrm{c}}$ & $0.34 \pm 0.04^{\mathrm{c}}$ \\
$\mathrm{B}$ & $0.38 \pm 0.09^{\mathrm{c}}$ & $0.04 \pm 0.01^{\mathrm{c}}$ & $0.07 \pm 0.02^{\mathrm{c}}$ & $0.04 \pm 0.01^{\mathrm{c}}$ & $0.15 \pm 0.04^{\mathrm{c}}$ \\
$\mathrm{C}$ & $0.80 \pm 0.06^{\mathrm{c}}$ & $0.08 \pm 0.01^{\mathrm{c}}$ & $0.13 \pm 0.01^{\mathrm{c}}$ & $0.12 \pm 0.02^{\mathrm{c}}$ & $0.32 \pm 0.05^{\mathrm{c}}$ \\
$\mathrm{D}$ & $3.60 \pm 0.23^{\mathrm{a}}$ & $0.35 \pm 0.04^{\mathrm{a}}$ & $0.66 \pm 0.06^{\mathrm{a}}$ & $0.54 \pm 0.05^{\mathrm{a}}$ & $1.54 \pm 0.09^{\mathrm{a}}$ \\
E & $2.66 \pm 0.28^{\mathrm{b}}$ & $0.21 \pm 0.07^{\mathrm{b}}$ & $0.47 \pm 0.07^{\mathrm{b}}$ & $0.39 \pm 0.04^{\mathrm{b}}$ & $1.07 \pm 0.10^{\mathrm{b}}$ \\
L.S.D. $(\mathrm{p}<0.05)$ & 0.51 & 0.06 & 0.13 & 0.09 & 0.21 \\
\hline
\end{tabular}

Sites: A = Karachi University Campus; B = Indus Battery factory; C = Universal Chemicals Factory; $D=$ Haroon Textile Factory; $E=$ National Foods Ltd. Factory. Means followed by the same letter in the same column are not significantly different according to Duncan Multiple Range Test at $p<0.05$ level \pm Standard Error, L.S.D. Least Significant Difference.

Table 2. Percentage reduction in growth of Prosopis juliflora in soils of different factories in comparison with control soil.

\begin{tabular}{lccccccccccc}
\hline Treatments & $\begin{array}{c}\text { Root } \\
\text { length }\end{array}$ & $\begin{array}{c}\text { Shoot } \\
\text { length }\end{array}$ & $\begin{array}{c}\text { Seedling } \\
\text { length }\end{array}$ & $\begin{array}{c}\text { Plant } \\
\text { cover }\end{array}$ & $\begin{array}{c}\text { Number } \\
\text { of } \\
\text { leaves }\end{array}$ & $\begin{array}{c}\text { Leaf } \\
\text { area }\end{array}$ & $\begin{array}{c}\text { Seedling } \\
\text { fresh } \\
\text { weight }\end{array}$ & $\begin{array}{c}\text { Root } \\
\text { dry } \\
\text { weight }\end{array}$ & $\begin{array}{c}\text { Shoot } \\
\text { dry } \\
\text { weight }\end{array}$ & $\begin{array}{c}\text { Leaf } \\
\text { dry } \\
\text { weight }\end{array}$ & $\begin{array}{c}\text { Total } \\
\text { plant } \\
\text { dry } \\
\text { weight }\end{array}$ \\
\hline A & -44.43 & -32.22 & -39.67 & -16.06 & -52.52 & -33.05 & -47.94 & -50.00 & -56.25 & -63.64 & -55.88 \\
B & -9.22 & +4.72 & -2.28 & -2.24 & -2.16 & +43.22 & +9.59 & 00 & -18.75 & +9.09 & -5.88 \\
C & +39.82 & +110 & +74.71 & +69.30 & +16.43 & +181.36 & +393.16 & +337.5 & +312.5 & +390.91 & +352.94 \\
D & -2.89 & +76.11 & +36.42 & +59.50 & +90.65 & +101.69 & +264.38 & +162.5 & +193.75 & +256.41 & +214.71 \\
\hline
\end{tabular}

Symbol: + = Percentage increase. Sites: A = Indus Battery Factory; B = Universal Chemicals Factory; C = Haroon Textile Factory; D = National Foods Ltd. Factory.

shoot, leaf and total plant dry weights of $P$. juliflora were also recorded in soils of Karachi University Campus (Control) and different industries (Polluted). The effects of different soil types on fresh and dry weight of root, shoot, leaf and total plant dry weight, was recorded for $P$. juliflora (Table 1b). Results also clearly indicated that soil samples of Indus Battery Factory significantly decreased fresh and dry weigh of $P$. juliflora as compared to University Campus and other industrial sites of the study areas.

The percentage reduction in different growth parameters of $P$. juliflora as compared to control showed that all the growth variables were increased in the soils of
Haroon Textile and National Foods Ltd., while in Indus Battery the percentage was decreased. Percentage reduction in root, shoot, seedling length, number of leaves, leaf area and biomass of $P$. juliflora was maximum in soils of Indus Batter Factory followed by Universal Chemical Factory and the lowest in National Foods Ltd. in comparison with Karachi University Campus (control) soil. The increase in seedling growth variable of $P$. juliflora was recorded for Haroon Textile soil as compared to soils of other industries (Table 2).

The correlation coefficient between growth parameters and soil characteristics for each species are calculated. General range of value for correlation coefficient is 
Table 3. Physical characteristics of different soils.

\begin{tabular}{lccccccl}
\hline Locality & M.W.H.C. (\%) & B.D. $\left(\right.$ gcc $\left.^{-1}\right)$ & Porosity (\%) & Sand (\%) & Silt (\%) & Clay (\%) & Soil texture class \\
\hline A & $22.30 \pm 1.14^{\mathrm{a}}$ & $1.36 \pm 0.02^{\mathrm{ab}}$ & $49.0 \pm 2.82^{\mathrm{ab}}$ & $24.34 \pm 0.10^{\mathrm{a}}$ & $44.28 \pm 0.10^{\mathrm{c}}$ & $31.42 \pm 0.19^{\mathrm{d}}$ & Clay loam. \\
$\mathrm{B}$ & $28.91 \pm 0.21^{\mathrm{b}}$ & $1.27 \pm 0.11^{\mathrm{a}}$ & $52.0 \pm 1.41^{\mathrm{a}}$ & $29.30 \pm 0.15^{\mathrm{b}}$ & $47.00 \pm 0.01^{\mathrm{d}}$ & $23.70 \pm 0.14^{\mathrm{b}}$ & Silty loam \\
$\mathrm{C}$ & $23.12 \pm 0.09^{\mathrm{a}}$ & $1.55 \pm 0.11^{\mathrm{b}}$ & $41.5 \pm 0.7^{\mathrm{c}}$ & $38.80 \pm 0.16^{\mathrm{c}}$ & $30.50 \pm 0.19^{\mathrm{b}}$ & $30.70 \pm 0.56^{\mathrm{d}}$ & Sandy-loam \\
$\mathrm{D}$ & $23.88 \pm 0.24^{\mathrm{a}}$ & $1.46 \pm 0.09^{\mathrm{ab}}$ & $45.0 \pm 2.82^{\mathrm{bc}}$ & $59.80 \pm 1.38^{\mathrm{d}}$ & $13.00 \pm 0.71^{\mathrm{a}}$ & $27.20 \pm 0.64^{\mathrm{c}}$ & Sandy clay loam \\
E & $23.91 \pm 0.21^{\mathrm{a}}$ & $1.54 \pm 0.05^{\mathrm{b}}$ & $42.0 \pm 1.40^{\mathrm{c}}$ & $69.44 \pm 0.44^{\mathrm{e}}$ & $11.00 \pm 1.0^{\mathrm{a}}$ & $19.56 \pm 0.10^{\mathrm{a}}$ & Sandy loam \\
L.S.D. (p<0.05) & 1.91 & 0.23 & 5.21 & 2.39 & 2.03 & 1.45 & - \\
\hline
\end{tabular}

Sites: $A=$ Karachi University Campus; $B=$ Indus Battery factory; $C=$ Universal Chemicals factory; $D=$ Haroon Textile factory; $E=$ National Foods Ltd. factory. Symbol: M.W.H.C. = Maximum Water Holding Capacity; B.D. = Bulk Density \pm Standard Error. Source: Kabir (2014).

Abbreviation: $\mathbf{C a C O}_{3}=$ Calcium Carbonate; $\mathbf{C l}=$ Chloride; $\mathbf{p H}=$ Power of Hydrogen ion; O.M. = Organic Matter; T.O.C. = Total Organic Carbon; $\mathbf{S}=$ Sulphur; E.C. = Electrical Conductivity; T.D.S. = Total Dissolved Salts; $\mathbf{N a}=$ Sodium; $\mathbf{K}=$ Potassium. Means followed by the same letter in the same column are not significantly different according to Duncan Multiple Range Test at $p<0.05$ level. \pm Standard Error, L.S.D. Least Significant Difference.

Table 4. Chemical characteristics of different soils.

\begin{tabular}{|c|c|c|c|c|c|c|c|c|c|c|}
\hline \multirow{2}{*}{ Locality } & \multirow{2}{*}{$\mathrm{CaCO}_{3}(\%)$} & \multirow{2}{*}{$\mathrm{Cl}\left(\mathrm{mgL}^{-1}\right)$} & \multirow{2}{*}{$\mathrm{pH}$} & \multirow{2}{*}{ O.M. (\%) } & \multirow{2}{*}{ T.O.C. (g) } & \multirow{2}{*}{$S\left(\mu g g^{-1}\right)$} & \multirow{2}{*}{ E.C. $\left(\mathrm{dScm}^{-1}\right)$} & \multirow{2}{*}{ T.D.S. $\left(\mathrm{mgL}^{-1}\right)$} & \multicolumn{2}{|c|}{ Exchangeable } \\
\hline & & & & & & & & & $\mathrm{Na}(\mathrm{ppm})$ & $\mathrm{K}$ (ppm) \\
\hline$A$ & $21.60 \pm 1.2^{b}$ & $00 \pm 0.0^{a}$ & $7.00 \pm 0.15^{b}$ & $4.50 \pm 0.28^{a}$ & $2.61 \pm 0.03^{e}$ & $58.75 \pm 2.86^{b}$ & $19.00 \pm 0.3^{d}$ & $13.90 \pm 0.7^{d}$ & $190 \pm 10^{b}$ & $156 \pm 4^{b}$ \\
\hline B & $31.65 \pm 0.27^{\mathrm{C}}$ & $00 \pm 0.0^{a}$ & $6.54 \pm 0.06^{a}$ & $7.56 \pm 0.10^{b}$ & $4.38 \pm 0.02^{d}$ & $41.25 \pm 0.12^{\mathrm{a}}$ & $33.20 \pm 0.6^{\mathrm{e}}$ & $24.50 \pm 0.3^{\mathrm{e}}$ & $410 \pm 10^{c}$ & $162 \pm 6^{b}$ \\
\hline C & $14.15 \pm 0.31^{\mathrm{a}}$ & $400 \pm 10^{c}$ & $6.81 \pm 0.05^{\mathrm{ab}}$ & $3.23 \pm 0.12^{\mathrm{a}}$ & $1.87 \pm 0.04^{c}$ & $40.00 \pm 3.0^{a}$ & $7.20 \pm 0.30^{b}$ & $5.20 \pm 0.30^{b}$ & $650 \pm 10^{e}$ & $197 \pm 6^{c}$ \\
\hline D & $19.75 \pm 0.09^{b}$ & $710 \pm 25^{d}$ & $6.66 \pm 0.03^{a}$ & $3.26 \pm 0.09^{a}$ & $1.89 \pm 0.01^{\mathrm{a}}$ & $45.00 \pm 3.0^{a}$ & $9.60 \pm 0.20^{c}$ & $7.10 \pm 0.10^{c}$ & $567 \pm 7.0^{d}$ & $207 \pm 8^{c}$ \\
\hline$E$ & $19.55 \pm 0.18^{b}$ & $140 \pm 5.0^{b}$ & $7.65 \pm 0.06^{c}$ & $3.42 \pm 0.08^{a}$ & $1.98 \pm 0.01^{b}$ & $125.00 \pm 4.0^{c}$ & $0.80 \pm 0.10^{a}$ & $0.60 \pm 0.10^{a}$ & $113 \pm 7.0^{\mathrm{a}}$ & $74 \pm 6^{a}$ \\
\hline L.S.D. $(p<0.05)$ & 2.08 & 44.52 & 0.30 & 0.56 & 0.09 & 10.56 & 1.25 & 1.35 & 32.43 & 22.29 \\
\hline
\end{tabular}

Sites: A = Karachi University Campus; $B=$ Indus Battery factory; $C=$ Universal Chemicals factory; $D=$ Haroon Textile factory; $E=$ National Foods Ltd. Factory.

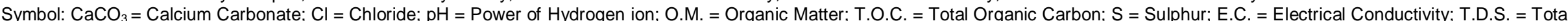

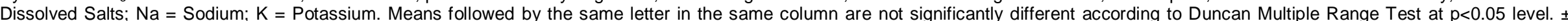
Standard Error, L.S.D. Least Significant Difference. Source: Kabir (2014).

summarized in Table 4 to 6 and found that some of physical soil characteristics were significantly correlated with growth parameters at $p<0.01$ and $p<0.05$. Sand was highly positive correlated while, maximum water holding capacity and clay showed non-significant correlation with different growth parameters of $P$. juliflora (Table 5). In chemical soil characteristics, chloride was highly positive correlated while electrical conductivity and total dissolved salts are highly negative correlated with different growth parameters at $p<$ 0.01 significant level. Calcium carbonate, $\mathrm{pH}, \mathrm{Na}$ and $\mathrm{K}$ showed non-significant correlation with different growth parameters of $P$. juliflora (Table 6 ). Cross correlation among growth parameters and soil characteristics were also determined for $P$. juliflora. All growth parameters were significant $(p<0.05)$ and highly positive correlated at significant level $p<0.01$ while, cross correlation among soil characteristics was non-significant, highly positive as well as highly negative correlated at $p<0.05$ and $p<0.01$ significant levels (Table 7).

The variation in seedling growth $P$. juliflora growing in soils of different area in pot conditions before and after harvest is shown in Figure 1. The seedling size and number of leaves increased in 
Table 5. Pearson's correlation of soil physical characteristics and growth parameters of Prosopis juliflora.

\begin{tabular}{|c|c|c|c|c|c|c|}
\hline Growth parameters & M.W.H.C. & B.D. & Porosity & Sand & Silt & Clay \\
\hline Root length & $-0.480^{*}$ & 0.349 & -0.338 & 0.388 & $-0.497^{*}$ & 0.162 \\
\hline Shoot length & -0.321 & $0.456^{*}$ & $-0.451^{*}$ & $0.694^{* *}$ & $-0.736^{\star *}$ & -0.231 \\
\hline Seedling size & $-0.424^{*}$ & $0.465^{*}$ & $-0.456^{*}$ & $0.643^{\star *}$ & $-0.716^{* *}$ & -0.095 \\
\hline Plant cover & -0.334 & $0.539^{* *}$ & $-0.532^{\star \star}$ & $0.911^{* *}$ & $-0.931^{* *}$ & $-0.421^{*}$ \\
\hline Number of leaves & -0.325 & $0.416^{*}$ & $-0.410^{*}$ & $0.653^{* *}$ & $-0.704^{* *}$ & -0.177 \\
\hline Leaf area & -0.344 & $0.529^{* *}$ & $-0.526^{\star *}$ & $0.721^{* *}$ & $-0.789^{\star *}$ & -0.156 \\
\hline Seedling fresh weight & -0.278 & $0.477^{*}$ & $-0.473^{*}$ & $0.853^{\star *}$ & $-0.885^{\star \star}$ & -0.349 \\
\hline Root dry weight & -0.262 & 0.396 & -0.391 & $0.765^{\star *}$ & $-0.811^{* *}$ & -0.254 \\
\hline Shoot dry weight & -0.278 & $0.435^{*}$ & $-0.430^{*}$ & $0.805^{* *}$ & $-0.839^{* *}$ & -0.313 \\
\hline Leaf dry weight & -0.306 & $0.480^{*}$ & $-0.475^{\star}$ & $0.824^{\star *}$ & $-0.864^{\star *}$ & -0.304 \\
\hline Total plant dry weight & -0.295 & $0.455^{\star}$ & $-0.450^{*}$ & $0.826^{* *}$ & $-0.867^{\star *}$ & -0.305 \\
\hline
\end{tabular}

${ }^{*},{ }^{* *}$ Correlation is significant at the $p<0.05$ and $p<0.01$ levels, respectively (2-tailed)

Abbreviation: M.W.H.C. = Maximum Water Holding Capacity; B.D. = Bulk Density.

Table 6. Pearson's correlation of soil chemical characteristics and growth parameters of Prosopis juliflora.

\begin{tabular}{|c|c|c|c|c|c|c|c|c|c|c|}
\hline Growth parameters & $\mathrm{CaCO}_{3}$ & $\mathrm{Cl}$ & $\mathrm{pH}$ & O.M. & T.O.C. & $\mathbf{S}$ & E.C. & T.D.S. & $\mathrm{Na}$ & $\mathrm{K}$ \\
\hline Root length & $-0.422^{*}$ & $0.559^{* *}$ & 0.076 & $-0.560^{\star *}$ & $-0.560^{* *}$ & 0.044 & $-0.443^{*}$ & $-0.442^{*}$ & 0.126 & 0.211 \\
\hline Shoot length & -0.353 & $0.558^{* *}$ & 0.289 & $-0.553^{\star *}$ & $-0.553^{* *}$ & & & $-0.562^{* *}$ & & -0.072 \\
\hline Seedling size & $-0.422^{*}$ & 0.61 & 0.237 & -0.6 & & 247 & ** & $-0.576^{\star \star}$ & & 0.032 \\
\hline Plant cover & -0.361 & $0.601^{* *}$ & $0.450^{*}$ & $-0.632^{\star *}$ & $-0.632^{\star *}$ & $0.518^{\star \star}$ & $-0.691^{* *}$ & $-0.687^{\star \star}$ & -0.122 & -0.230 \\
\hline Number of $\mathrm{l} \epsilon$ & -0.338 & $0.578^{* *}$ & 0.229 & $-0.540^{\star *}$ & $-0.540^{\star *}$ & 0.263 & $-0.527^{* *}$ & $-0.524^{\star *}$ & 0.030 & -0.011 \\
\hline Leaf area & $-0.440^{*}$ & $0.717^{* *}$ & 0.190 & $-0.625^{\star *}$ & $-0.625^{\star *}$ & 0.212 & $-0.609^{* *}$ & $-0.606^{\star \star}$ & 0.176 & 0.071 \\
\hline Seedling fre & -0.324 & $0.676^{* *}$ & 0.297 & $-0.585^{\star *}$ & $-0.585^{\star *}$ & 0.371 & $-0.612^{* *}$ & $-0.607^{\star *}$ & 0.007 & -0.089 \\
\hline Root dry weight & -0.287 & $0.702^{* *}$ & 0.176 & $-0.541^{\star *}$ & $-0.541^{* *}$ & 0.250 & $-0.527^{* *}$ & $-0.523^{\star *}$ & 0.075 & 0.026 \\
\hline Shoot dry weight & -0.302 & $0.653^{* *}$ & 0.270 & $-0.560^{\star *}$ & $-0.560^{\star *}$ & 0.342 & $-0.573^{* *}$ & $-0.569^{\star *}$ & 0.001 & -0.068 \\
\hline Leaf dry weight & -0.347 & $0.678^{* *}$ & 0.284 & $-0.598^{* *}$ & $-0.598^{* *}$ & 0.347 & $-0.612^{* *}$ & $-0.608^{\star *}$ & 0.022 & -0.062 \\
\hline Total plant dry weight & -0.324 & $0.692^{* *}$ & 0.263 & $-0.587^{* *}$ & $-0.587^{* *}$ & 0.334 & $-0.594^{* *}$ & $-0.590^{* *}$ & 0.024 & -0.047 \\
\hline
\end{tabular}

${ }^{*},{ }^{* *}$ Correlation is significant at the $p<0.05$ and $p<0.01$ levels, respectively (2-tailed).

Abbreviation and symbol: $\mathrm{CaCO}_{3}=$ Calcium Carbonate; $\mathrm{Cl}=$ Chloride; $\mathrm{pH}=$ Power of Hydrogen ion; O.M. = Organic Matter; T.O.C. $=$ Total Organic Carbon; $\mathrm{S}=$ Sulphur; E.C. = Electrical Conductivity; T.D.S. = Total Dissolved Salts; $\mathrm{Na}=$ Sodium; $\mathrm{K}=$ Potassium.

National Foods Ltd., during $1^{\text {st }}$ week and maintained it for rest of the growth period. The periodical growth of $P$. juliflora revealed that seedling size, number of leaves and plant circumference were increased with time but this increase was more prominent for seedlings raised in soil of National Foods Ltd. and Haroon Textile factories. The seedling size, number of leaves and plant circumference were reduced in soil of Indus Battery factory (Figure 2). The effect of non-industrial area soil on plant height, plant cover and number of leaves of $P$. juliflora periodically on eight weeks was found less affected as compared to soil of Indus Battery Factory treatment.

\section{DISCUSSION}

Seed germination and seedling growth are two important and vulnerable stages in the early life cycle of terrestrial angiosperms and any kind of stress (Naik et al., 2015). The rapid industrial growth and discharge of various forms of pollutants from industries without any proper treatment are causing serious pollution problems in the Sindh Industrial Trading Estate (S.I.T.E.), Karachi. The results of the present study indicated that the soil of study area was disturbed due to pollutants released from different industries. Toxic nature of these pollutants varies significantly from industry to industry affecting growth of plant species. Reduction trend in different growth parameters was not the same but varies from industry to industry and were recorded more for most of the species grown in soils of Indus Battery and Universal Chemicals factories. The harmful industrial waste and effluent are steadily increasing in current years and their discharge on soil, canals, rivers and water course causes serious environmental related problems for normal growth of flora and fauna of the region (Kashem and 
Table 7. Pearson's cross correlations among growth parameters of Prosopis juliflora.

\begin{tabular}{|c|c|c|c|c|c|c|c|c|c|c|c|}
\hline Growth parameters & $\begin{array}{l}\text { Root } \\
\text { length }\end{array}$ & $\begin{array}{l}\text { Shoot } \\
\text { length }\end{array}$ & $\begin{array}{l}\text { Seedling } \\
\text { length }\end{array}$ & $\begin{array}{l}\text { Plant } \\
\text { cover }\end{array}$ & $\begin{array}{c}\text { Number of } \\
\text { leaves }\end{array}$ & Leaf area & $\begin{array}{l}\text { Seedling } \\
\text { fresh wt. }\end{array}$ & Root dry wt. & Shoot dry wt. & $\begin{array}{l}\text { Leaf dry } \\
\text { wt. }\end{array}$ & $\begin{array}{c}\text { Total plant } \\
\text { dry wt. }\end{array}$ \\
\hline Root length & 1 & & & & & & & & & & \\
\hline Shoot length & $0.624^{* *}$ & 1 & & & & & & & & & \\
\hline Seedling length & $0.835^{\star *}$ & $0.951^{* *}$ & 1 & & & & & & & & \\
\hline Plant cover & $0.525^{\star *}$ & $0.788^{\star \star}$ & $0.764^{\star *}$ & 1 & & & & & & & \\
\hline Number of leaves & $0.606^{* *}$ & $0.822^{* *}$ & $0.819^{* *}$ & $0.764^{* *}$ & 1 & & & & & & \\
\hline Leaf area & $0.582^{* *}$ & $0.835^{\star *}$ & $0.818^{* *}$ & $0.816^{\star *}$ & $0.814^{\star *}$ & 1 & & & & & \\
\hline Seedling fresh wt. & $0.637^{* *}$ & $0.863^{\star *}$ & $0.859^{\star *}$ & $0.946^{\star *}$ & $0.867^{\star *}$ & $0.858^{* *}$ & 1 & & & & \\
\hline Root dry wt. & $0.768^{* *}$ & $0.764^{\star *}$ & $0.841^{* *}$ & $0.871^{* *}$ & $0.713^{\star *}$ & $0.758^{* *}$ & $0.927^{* *}$ & 1 & & & \\
\hline Shoot dry wt. & $0.650^{* *}$ & $0.925^{\star *}$ & $0.907^{\star *}$ & $0.920^{* *}$ & $0.873^{\star *}$ & $0.856^{\star *}$ & $0.980^{\star *}$ & $0.902^{* *}$ & 1 & & \\
\hline Leaf dry wt. & $0.643^{\star *}$ & $0.789^{\star *}$ & $0.810^{\star *}$ & $0.917^{* *}$ & $0.886^{\star *}$ & $0.873^{* *}$ & $0.968^{* *}$ & $0.905^{\star *}$ & $0.923^{* *}$ & 1 & \\
\hline Total plant dry wt. & $0.693^{* *}$ & $0.866^{\star *}$ & $0.884^{* *}$ & $0.935^{\star *}$ & $0.868^{* *}$ & $0.866^{* *}$ & $0.993^{* *}$ & $0.951^{* *}$ & $0.979^{* *}$ & $0.974^{* *}$ & 1 \\
\hline
\end{tabular}

* ${ }^{* *}$ Correlation is significant at the $p<0.05$ and $p<0.01$ levels, respectively (2-tailed) Abbreviation: wt. $=$ Weight.

Singh, 1998). In soils of Indus Battery and Universal Chemicals factories, low nutrients supply and toxic effects of discharged pollutants inhibited growth of different plant species. The seedling length of $P$. juliflora followed the similar pattern of reduction in shoot as well as root length because root and shoot are exposed to either direct or indirect to different industrial pollutant discharges in air, water and soil. Iqbal and Shazia (2004) recorded the related decreased in root shoot and seedling length along with seedling dry weight of Albizia lebbeck and Leucaena leucocephala by the use of different levels of lead $(\mathrm{Pb})$ and cadmium $(\mathrm{Cd})$. In $P$. juliflora, a significant increase in seedling growth variable was recorded which may be the result of their tolerance for pollutants discharged from Haroon Textile and National Foods Factory Ltd as compared to Indus Battery Factory, Universal Chemical Factory soils and Karachi University Campus soil. The sensitivity to different pollutants differs between plants and even between clones of the same species (Pukacka and Pukacki, 2000). The seedling growth of $P$. juliflora showed that this species adopted best in soils of Haroon Textile and National Foods Factory Ltd. while in Indus Battery Factory and Universal Chemical Factory soils its growth was reduced as compared to Karachi University Campus soil. The variation in seedling growth of different plant species may be related with the amounts of industrial pollutants. This is related to the fact that some fundamental nutrients present in the industrial effluents are essentials but above a critical concentration, they become harmful. As growth of $P$. juliflora was found better in soil of National Foods Ltd., which may be the result of more nutrient supply in soil that are being used to manufacture different food products. Similar findings were reported from the study of Panaskar and Pawar (2011a, b) which proved that textile pollutants were not inhibitory at low concentrations above a particular concentration growth and development of seedlings was affected.
The soil of S.I.T.E. area is constantly absorbing, accumulating the industrial pollutants, which are continuously incorporating into ecosystems. It was reported that depending on their resistant or sensitivity level, plants showed visible changes along with reduction in different growth parameters. As industrial area plants are facing different challenges which would comprise on variation in the biochemical processes such as total chlorophyll and carotenoid or accumulation of certain metabolites (Agbaire and Esiefarienrhe, 2009). Extreme quantity of toxic industrial pollutants regularly caused declined in plant growth and bio-chemical activities (Prodgers and Inskeep, 1981).

From different parts of plant, the leaf is a most receptive part to be influenced by industrial pollutants. In our study, all leaf related parameters which include number of leaf, leaf area and leaf dry weight were reduced in seedlings raised from soil of Indus Battery and Universal Chemicals factories. The sensitivity is dependent on the 

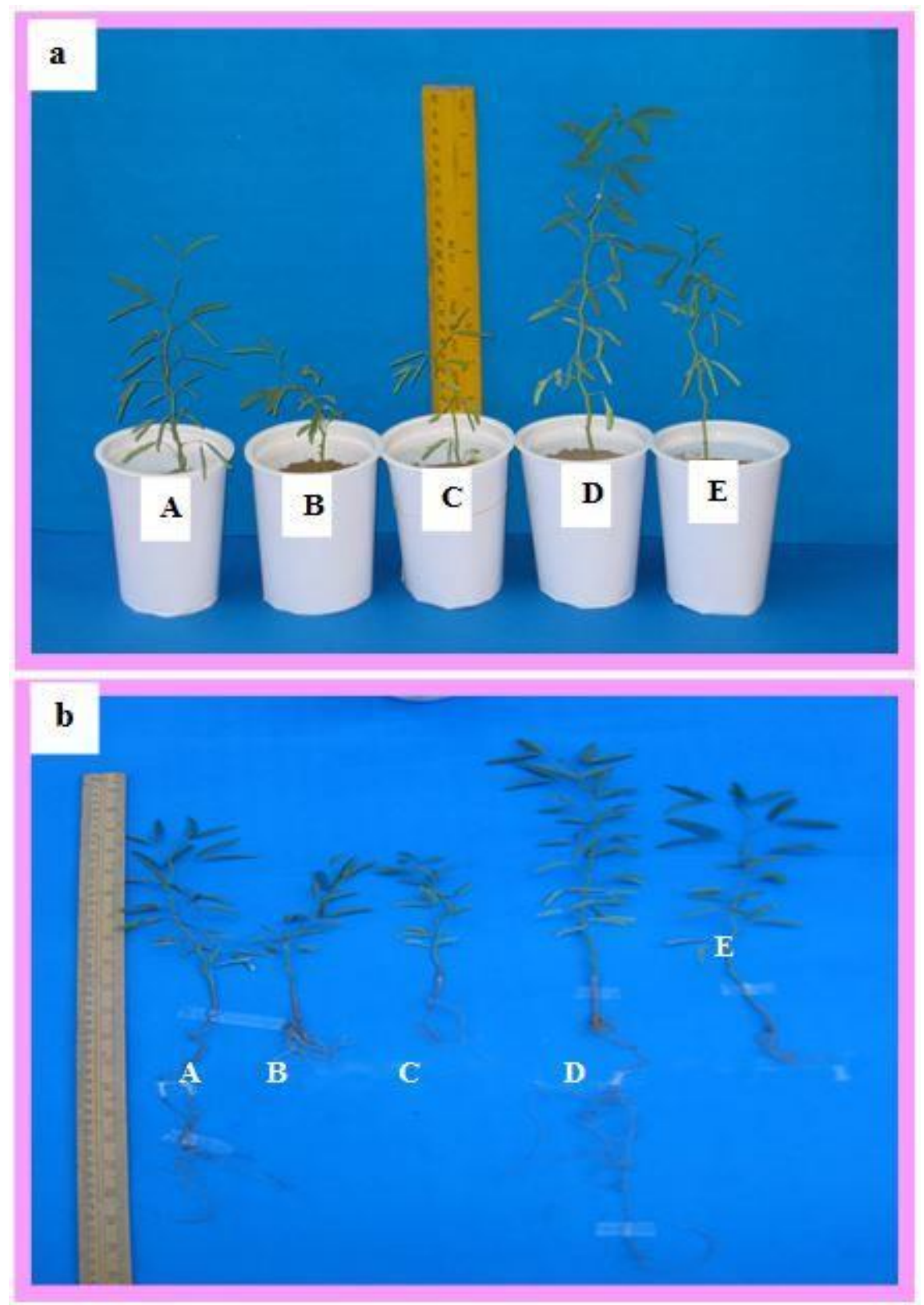

Figure 1. Growth of Prosopis juliflora in different soils (a) and after ten week of harvest (b). Sites: $\mathbf{A}=$ Karachi University Campus soil, $\mathbf{B}=$ Indus Battery Factory soil, $\mathbf{C}=$ Universal Chemicals Factory soil, $\mathbf{D}=$ Haroon Textile Factory soil, $\mathbf{E}=$ National Foods Ltd. Factory soil.

reality that the major portion of the fundamental physiological activities such as photosynthesis and gaseous exchange are occurring in the leaf. So, the leaf at its different phases of growth and development, acts as a best indicator to different industrial contaminants (Shafiq et al., 2009). Reduction in leaf concerned parameters of seedlings raised in soils of industrial area was the witness of toxic effects of the industrial polluted environment. It was recorded that the plants grown in soils of Indus Battery and Universal Chemicals Factories.
The inhibition of the growth and developmental phases of sensitive plants are resulting the existence of toxic substance in the industrial pollutants. The reduced leaf area, leaf fresh and dry weights is directly interrelated to different kinds of toxic industrial pollutants which are being discharged into the ecosystem by different types of industries. These findings are supported by the efforts of Sibak and Gulyas (1990), lqbal and Shafiq (2000) and Shafiq and lqbal $(2003,2005)$ who recorded reduction in leaf size due to air carry pollutants resulting from 

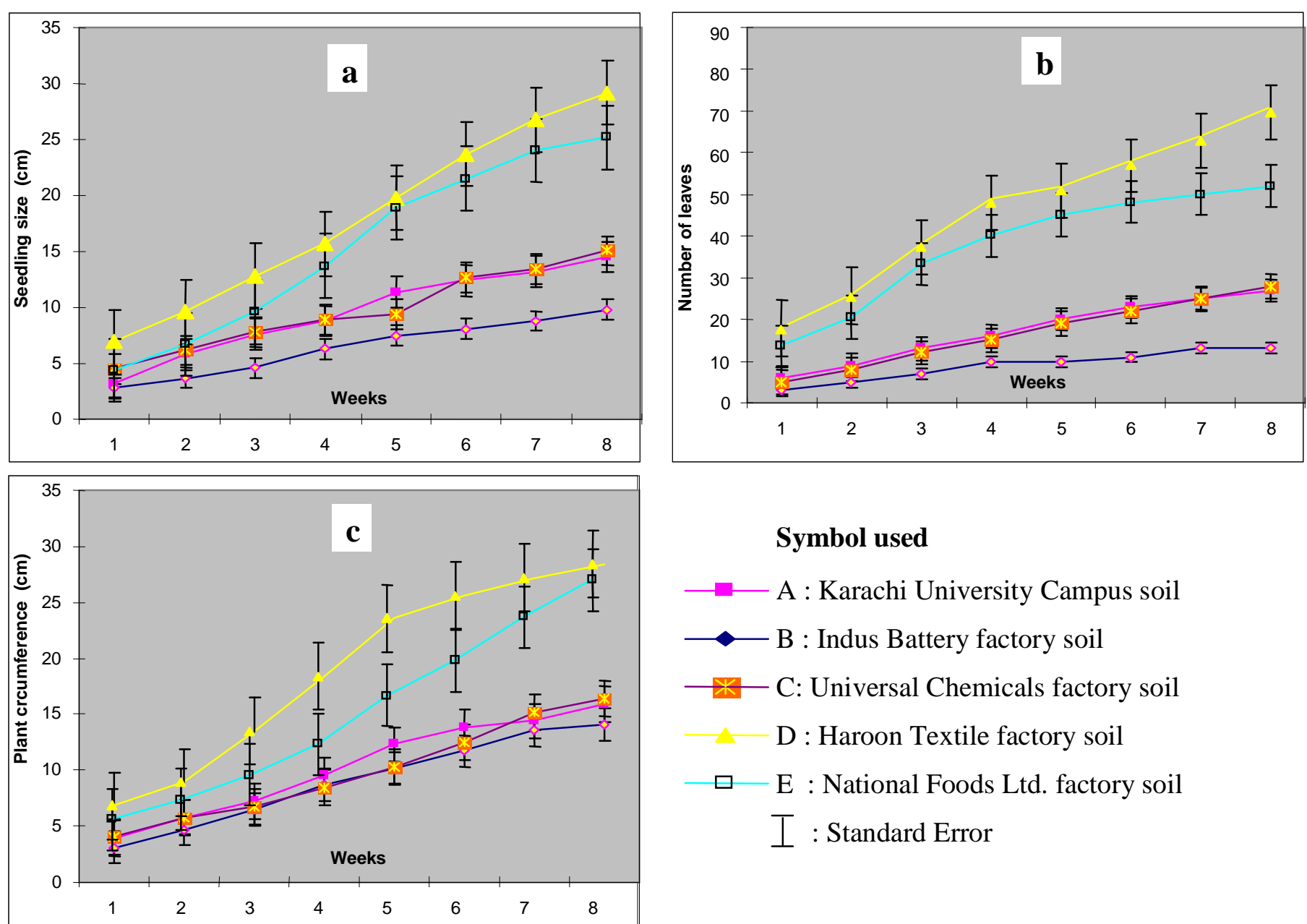

\section{Symbol used}

—— A : Karachi University Campus soil

$\longrightarrow$ B : Indus Battery factory soil

世-C: Universal Chemicals factory soil

D : Haroon Textile factory soil

$\square \quad$ E : National Foods Ltd. factory soil

I : Standard Error

Figure 2. Seedling size (a), number of leaves (b) and plant circumference (c) of Prosopis juliflora growing in soils of different areas.

automobiles especially when carrying diverse industrial goods. Industrial estates in Karachi also have large quantity of automobiles for transport of different industrial products. Both increase and decrease in the weight related parameters were also described by Nawaz et al. (2006) and Yousaf et al. (2010).

The plants growth extensively related with soil physical and chemical characteristics such as water holding capacity of soil, bulk density, porosity and soil texture, that is, percentage of coarse sand, silt and clay while most common chemical properties include calcium carbonate, chloride, $\mathrm{pH}$, organic matter, total organic carbon, available soil sulfate, electrical conductivity and concentration of different salts (Kabir, 2014). Both an increase and decrease in these soil attributes showed considerable impacts on the growth of $P$. juliflora. Edaphic factors of S.I.T.E. industrial areas affected the seedling growth of $P$. juliflora especially in soils of Indus Battery and Universal Chemical factories, while an increase in growths of these species were observed in seedlings raised in soils of Haroon Textile and National
Foods Limited.

The Karachi University Campus soil with low maximum water holding capacity $(22.30 \%)$ might be the reason of not supporting the seedling growth performance of $P$. juliflora as compared to Haroon Textile and National Foods Ltd. The soils of Haroon Textile and National Foods Ltd., contained appreciable water holding capacity, bulk density and porosity as compared to control which might cause better seedling growth of $P$. juliflora. High soil $\mathrm{pH}$ was recorded for Haroon Textile and National Foods Ltd. Factory soil which decreased the translocation of pollutants including heavy metals into plants and resulted in their rapid growth of different plant species. As in soil of National Foods Ltd. Factory and Haroon Textile showed better growth even comparatively to soil of the Karachi University Campus. $\mathrm{pH}$ is an important factor which has an effect on the uptake and the transport of different heavy metals in soil. As soil pH is an essential parameter, regulating the accessibility of different metals to plants. Singh (1986) reported that the plant communities which had a high amount of soil organic 
matter, the maximum water holding capacity of soil was accordingly improved due to the colloidal nature of the organic matter. Sulfur is mostly available to plants in the form of sulfate in soil and the sulfur requirement is satisfied by its absorption through the root system (Herschbach, 2003; Herschbach et al., 2005). High electrical conductivity of Indus Battery factory soil may be the causative agent for reduction of most of growth parameters of different species. Electrical conductivity (E.C.) of soil is directly affected by the soils water contents, texture and proportion of soluble salts. Soil of Indus Battery factory has relatively high contents of electrical conductivity and total dissolved salts as compared to soils of other industries as well as Karachi University Campus which may be responsible for reduction in different growth parameters. High sodium and potassium contents in industrial soil might be another causative agent of reduction the seedling growth of $P$. juliflora, which could not adjust well in soil of Indus Battery and Universal Chemicals factories. Sodium usually plays a major role in salinity, as it is a component of the harmful salt sodium chloride. Low amount of total dissolved salts and calcium carbonate might also be responsible for enhancement of growth in plants. Because when soil contains high amount of soluble salts, then it develops unfavorable characters due to salinity which reduces the ability of plants to take up water, and this quickly causes reductions in growth rate, along with a suite of metabolic changes (Munns, 2002). The effects of high salts on plants are well known which reduced transpiration and result in growth retardation. The results indicated that root growth of the studied species was the most sensitive end point measurement than the shoot growth. As roots growth of $P$. juliflora not showed their better growth in seedlings raised in soils of Indus Battery and Universal Chemicals soils which resulted in their reduced growth. From the correlation interpretation of the obtained data, it is concluded that beside predominantly manifestations of interactions between different industrial pollutants and seedling growth, there is a common series of expression, as a general response to the stress caused by pollutants aggression, regardless of their toxic nature. It is evident from bar graph that $P$. juliflora seedlings followed same pattern of toxic index which increased from one to another factory. The different sensitive species can be used as bio-indicators and tolerant species can be used as a sink for industrial pollutants. It is clear that the industrial pollutants including lead battery manufacturer, chemicals, textile and foodstuffs are an operative ecological issue causing deterioration in the quality of Karachi's environment.

\section{Conclusion}

The seedling growth of $P$. juliflora showed well adapted in soils of Haroon Textile and National Foods Factory Ltd. while in Indus Battery Factory and Universal Chemical
Factory soils its growth was significantly $(p<0.05)$ reduced as compared to Karachi University Campus soil. This species may be planted around textile and foods industries due to its tolerance against the industrial activities. Furthermore, the relationship between five different soil types and seedlings of $P$. juliflora which were grown in soils of Indus Battery Factory, Universal Chemicals Factory, Haroon Textile Factory and National Foods Ltd. Factory showed significant $(p<0.05)$ variation in growth variables like root, shoot, seedling length, plant cover, leaf area, root, shoot and leaf dry weight and total plant dry weight. The observed results would be helpful in monitoring the impact of different soil types on the growth performance of plants and for the selection of plant species for cultivation and landscaping in such types of affected areas.

\section{REFERENCES}

Abdel SMF, Aly RO, 2000. Bioremediation of heavy metal contaminated soils in dry land. Pub. Marcel Dekkr, Inc. New York. 39: 677-692.

Agbaire PO, Esiefarienrhe E, 2009. Air pollution tolerance indices (APTI) of some plants around Otorogun gas plant in Delta State, Nigeria. J Appl Sci Environ Manag, 13: 11-14.

Alban L, Matorel M, Romero J, Grados N, Cruz G, Felker P, 2002. Cloning of elite, multipurpose trees of the Prosopis juliflora/pallida complex in Piura, Peru. Agroforestry Syst, 54: 173-182.

Cunningham SD, Shann JR, Crowley DE, Anderson TA. 1997. Phytoremediation of contaminated water and soil. Soil and water contaminate, American Chemical Society Washington, DC, 2-17.

Gardea-Torresdey JL, Polette S, Arteaga KJ, Tiemann JB, Ganzalez $\mathrm{JH}, 1996$. Determination of the content of hazardous heavy metals on Laree tridentata grown around a contaminated area. Proceeding of Eleventh EPP conf. on Hazardous waste Research, Edited by Erickson. pp. 660.

Grigalaviciene I, Rutkovience V, Marozas V, 2005. The accumulation of heavy metals lead, copper and cadmium at roadside forest soil. Pol J Environ Study, 14: 109-115.

Herschbach C, 2003. Whole plant regulation of sulphur nutrition: Influences of the environment. Plant Biol, 5: 233-244.

Herschbach C, Mult S, Kreuzwieser J, Kopriva S, 2005. Influence of anoxia on whole plant sulphur nutrition of flooding tolerant poplar (Populus tremula $\times$ P. alba). Plant Cell Environ, 28: 167-175.

Iqbal MZ, Shafiq M, 2000. Periodical effects of automobile pollution on the growth of some roadside trees. Ecologia (Bratislava), 19: 104110.

Iqbal MZ, Shazia Y, 2004. Differential tolerance of Albizia lebbeck and Leucaena leucocephala at toxic levels of lead and cadmium. Pol J Environ Study, 13: 439-442.

Kabir M, 2014. Effects of industrial pollution on tolerance and distribution of plants. Ph. D. thesis. University of Karachi, Karachi, Pakistan.

Kaushik N, Kumar V, 2003. Khejri (Prosopis cineraria) based agroforestry system for arid Haryana, India. J Arid Environ, 55: 433440.

Lenacuru $\mathrm{Cl}$, 2003. Impacts of Prosopis species in Baringo district. Proceedings of workshop on integrated management of Prosopis species in Kenya, pp. 41-47.

Masilamani $P$, Vadivelu KK, 1997. Seed development and maturation in honey mesquite (Prosopis juliflora Swartz DC.). Bangladesh $J$ Forest Sci, 26: 68-73.

Munns R, 2002. Comparative physiology of salt and water stress. Plant Cell Environ, 25: 239-250.

Naheed S, Ariz N, Baig S, 1986. Studies of particulate air pollutants in the vicinity of a cement factory and its health hazards. Pak J Sci Ind Res, 29(4): 95-102. 
Naik MR, Barman D, Maruthi RT, Mandal UK, Kundu DK, 2015. Screening of Tossa jute (Corhcorus olitorius) varieties against salt stress. The Ecoscan, 9(3\&4): 815-819.

Nawaz S, Ali SM, Yasmin A, 2006. Effect of industrial effluents on seed germination and early growth of Cicer arientum. J Biosci, 6: 49-54.

Panaskar DB, Pawar RS, 2011a. Effect of textile mill effluent on growth of Vigna unguiculata and Pisum sativum seedlings. Indian J Sci Technol, 4: 266-272.

Panaskar DB, Pawar RS, 2011b. Effect of textile mill effluent on growth of Sorgham vulgare and Vigna aconitifolia seedlings. Indian J Sci Technol, 4: 273-278.

Pukacka S, Pukacki PM, 2000. Seasonal changes in antioxidant level of Scots pine (Pinus sylvestris L.) needles exposed to industrial pollution. Ascorbate and thiol content. Acta Physiologiae Plantarum, 22: 451-456.

Rehman AS, 2006. Effects of soil of industrial areas on plants. Ph.D. Thesis, Department of Botany, University of Karachi, Karachi, Pakistan. $161 \mathrm{pp}$.

Rehman AS, Iqbal MZ 2006. Seed germination and seedling growth of trees in soil extracts from Korangi and Landhi Industrial Areas of Karachi. J New Seeds, 8(4): 33-45.

Shafiq M, Iqbal M.Z, Kabir M, Farooqi ZR, 2019. Poison Land. Vegetation of disturbed and polluted areas in Pakistan. Strategic book publishing \& rights agency, U.S.A. $173 \mathrm{pp}$.

Shafiq M, Iqbal MZ, 2005. The toxicity effects of heavy metals on germination and seedling growth of Cassia siamea Lamark. J New Seeds, 7: 95-105.

Shafiq M, Iqbal MZ, 2012. Impact of Automobile Pollutants on Plants. LAMBERT Academic Publishing GmbH \& Co. KG Heinrich-BöckingStr. 6-8, 66121, Saarbrücken, Germany. 132 pp.

Shafiq M, Iqbal MZ, Athar M, Qayyum M, 2009. Effects of autoexhaust emission on the phenology of Cassia siamea and Peltophorum pterocarprum growing in different areas of Karachi. Afr J Biotechnol, 8: $2469-2475$

Shafiq M, Iqbal MZ. 2003. Effects of automobile pollution on the phenology and periodicity of some roadside plants. Pak J Bot, 35: 931-938

Sibak S, Gulyas S, 1990. Leaf anatomical changes in Perishing acaluous oak. Acta Biol, 36: 23-52.

Talpada PM, Shukla PC, 1988. Study on sugar and amino acids composition of Prosopis juliflora pods. Gujarat Agric Univ Res J, 14: 32-35.

Yousaf I, Ali SM, Yasmin A, 2010. Germination and early growth response of Glycine max varieties in textile and paper industry effluents. Pak J Bot, 42: 3857-3863.
Citation: Kabir M, Iqbal MZ, Shafiq M, Farooqi ZR, Habiba U, 2020. Seedling growth and development of Prosopis juliflora (Sw.) DC. in different soil types of industrial and non-industrial areas. Adv Med Plant Res, 8(1): 14-23. 